\title{
Main outcomes of the sudden cardiac arrest survey 2006 to 2016
}

\author{
Hajung Yoon, Yunhyung Kwon, Juyeon An, Sungok Hong, Young Taek Kim \\ Division of Chronic Disease Control, Korea Centers for Disease Control and Prevention, Cheongju, Korea
}

\section{STRUCTURE OF THE SUDDEN CARDIAC ARREST SURVEY}

The Korea Centers for Disease Control and Prevention (KCDC) performs various tasks related to public health and epidemiology, including the planning and administration of medical record surveys, assurance of data quality, standardization of survey questions and guidelines, analysis of results, production of main indices, and publication of statistics. The National Cardiac Arrest Survey and Monitoring Advisory Committee comprises specialists in related departments and academic institutions. This committee provides technical assistance and advice regarding the maintenance of a survey-monitoring system, development of questionnaires, and review and analysis of results with the intent to support policy development and stimulate research. These works are conducted in cooperation with the National Fire Agency, 17 "Si (cities)-Do (provinces)" Fire and Disaster Headquarter centers, and approximately 600 medical institutions. From the rescue activity records of the "Si-Do" Fire and Disaster Headquarters, which are provided by the National Fire Agency, the KCDC selects cases involving sudden cardiac arrest, standardizes the names of hospitals to which patients are transferred, and establishes a surveillance database. Subsequently, dedicated survey personnel employed by the KCDC visit approximately 600 hospitals identified as receiving transferred patients and survey the pertinent medical records. All involved hospitals cooperate actively with this work (Fig. 1).

\section{INCIDENCE OF SUDDEN CARDIAC ARREST AND SURVIVAL STATUS}

The number of cases of sudden cardiac arrest increased by 1.5-fold from 19,480 in 2006 to 29,832 in 2016, while the standardized incidence rate increased by 1.1-fold from 39.3 (per 100,000 population) to 41.5 during the same period (Fig. 2). The demographic features of cardiac arrest patients also changed with the overall demographic structure during the 11-year study period, as demonstrated by the sharp increase in the proportion of elderly patients (aged $\geq 70$ years) from 38.7\% in 2006 to $49.5 \%$ in 2016. Similarly, the proportion of arrests attributed to "disease" increased. The rates of survival and neurological recovery increased each year, with the former increasing by 3.3-fold from 2.3\% in 2006 to 7.6\% in 2016 (Fig. 3). Regarding the latter, the proportion of patients who neurologically recovered sufficiently to achieve independence with tasks of daily living increased by 7 -fold, from $0.6 \%$ in 2006 to $4.2 \%$ in 2016. Improved survival and neurological recovery also rely on the rate of cardiopulmonary resuscitation (CPR) performed by nonmedical personnel (i.e., a layperson). Notably, this rate increased significantly from 1.9\% in 2008 to $16.8 \%$ in 2016, and the annual trend continues to increase (Fig. 4). Furthermore, the rate of re-
elSSN: 2383-4625

Received: 16 April 2018

Revised: 12 September 2018

Accepted: 26 September 2018

Correspondence to: Young Taek Kim Division of Chronic Disease Control, Korea Centers for Disease Control and Prevention, 187 Osongsaengmyeong 2-ro, Osong-eup, Heungdeok-gu, Cheongju 28159, Korea

E-mail: ruyoung@korea.kr ORCID

http://orcid.org/0000-0002-6360-5950

This article is based on a study reported initially in "Korea Centers for Disease Control and Prevention. 2006-2016 Sudden Cardiac Arrest Statistics. Cheongju: Korea Centers for Disease Control and Prevention; 2017."

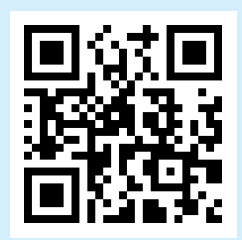

How to cite this article:

Yoon H, Kwon Y, An J, Hong S, Kim YT. Main outcomes of the sudden cardiac arrest survey 2006 to 2016. Clin Exp Emerg Med 2019;6(2):183-188.

This is an Open Access article distributed under the terms of the Creative Commons Attribution Non-Commercial License (http:// creativecommons.org/licenses/by-nc/4.0/). 

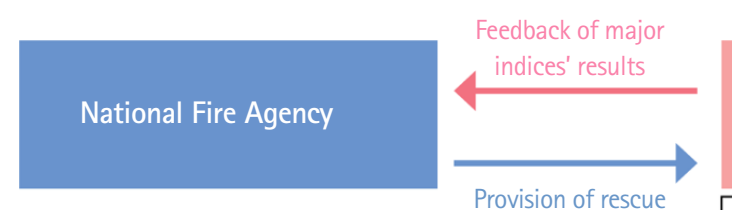

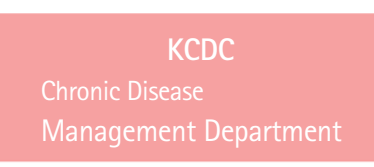

- Planning of program

- Carrying out surveys and data

registration

- Insuring quality of data

- Standardizing survey questions and guidelines

- Analysing and publishing statistical results

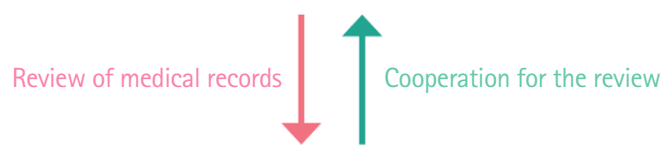

Medical institution
National Cardiac Arrest

Surveillence Advisory

Committee

- Advice on surveillence system

- Advice on survey question development

- Review of data analysis results

- Support and promotion of related policies' research and development

Fig. 1. Sudden cardiac arrest survey system. KCDC, Korea Centers for Disease Control and Prevention.

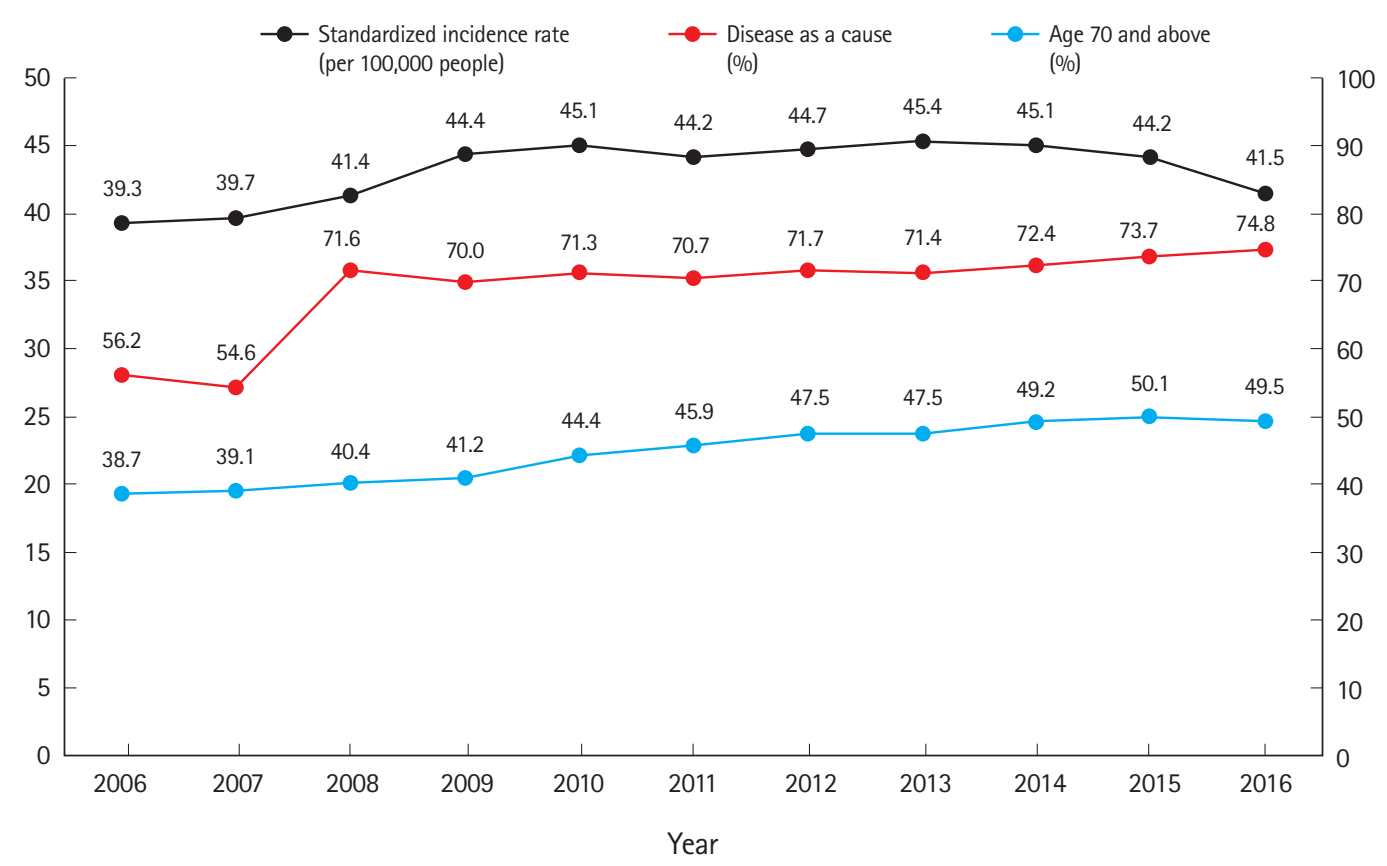

Fig. 2. Trends of sudden cardiac arrest.

turn of spontaneous circulation before hospital arrival, an indicator of the paramedics' capability, also increased by 7.7-fold from $0.9 \%$ in 2006 to $6.9 \%$ in 2016.

\section{STATUS OF SUDDEN CARDIAC ARRESTS IN EACH REGION}

Large disparities in the values of major indices associated with sudden cardiac arrest were observed among regions. Although the underlying cause remains unclear, the tendency of these disparities to increase highlights the need to produce relevant indices, and the data needs to be provided to the communities for local cooperation in the developement and implementation of effective policies. Notably, increases in regional disparities were observed both in the rate of CPR performed by a layperson and the rate of return of spontaneous circulation before hospital arrival, 
$(\%)$

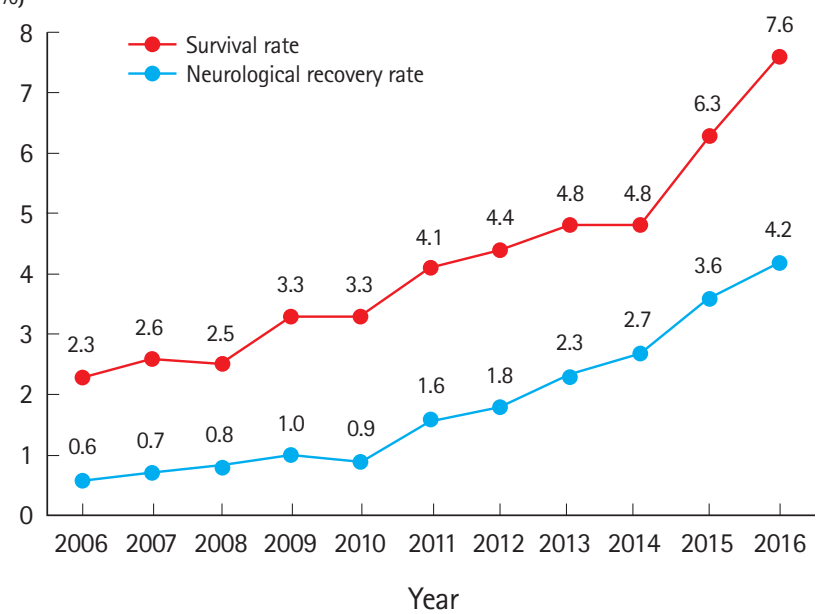

Fig. 3. Trends in survival rate and neurological recovery rate.

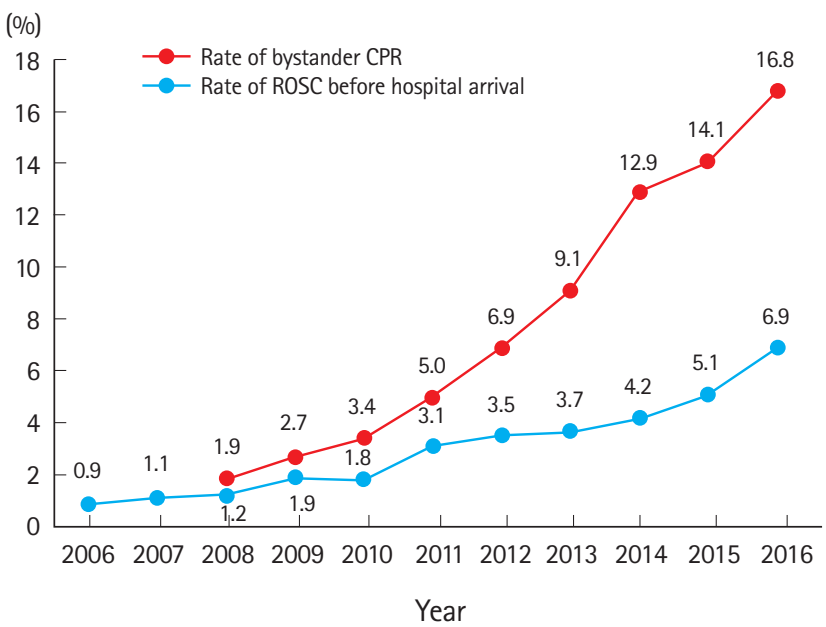

Fig. 4. Trends in rate of bystander cardiopulmonary resuscitation (CPR) and rate of return of spontaneous circulation (ROSC) before hospital arrival.

Table 1. Disparities in Sudden cardiac arrest survey main indices, 2006-2016

\begin{tabular}{|c|c|c|c|c|c|c|c|c|c|c|c|c|}
\hline Index & Value & 2006 & 2007 & 2008 & 2009 & 2010 & 2011 & 2012 & 2013 & 2014 & 2015 & 2016 \\
\hline \multirow{14}{*}{$\begin{array}{l}\text { Rate of CPR performed } \\
\text { by layperson ( } \%)\end{array}$} & Total & - & - & 1.9 & 2.7 & 3.4 & 5.0 & 6.9 & 9.1 & 12.9 & 14.1 & 16.8 \\
\hline & Seoul & - & - & 3.1 & 5.5 & 7.3 & 12.3 & 17.4 & 19.8 & 26.2 & 23.6 & 29.6 \\
\hline & Daegu & - & - & 1.6 & 3.0 & 4.1 & 5.5 & 6.1 & 9.6 & 12.9 & 18.3 & 23.6 \\
\hline & Incheon & - & - & 2.4 & 4.9 & 5.3 & 6.2 & 6.9 & 8.0 & 14.6 & 13.0 & 16.6 \\
\hline & Gwangju & - & - & 0.9 & 1.4 & 1.5 & 2.8 & 3.8 & 4.4 & 10.1 & 8.2 & 8.1 \\
\hline & Ulsan & - & - & 1.6 & 1.3 & 1.6 & 4.9 & 4.7 & 7.3 & 8.1 & 14.9 & 20.7 \\
\hline & Sejong & - & - & - & - & - & - & 3.8 & 2.6 & 2.6 & 7.1 & 13.4 \\
\hline & Gyeonggi & - & - & 2.2 & 2.7 & 4.0 & 5.1 & 6.8 & 8.5 & 12.6 & 16.3 & 18.9 \\
\hline & Gangwon & - & - & 2.1 & 2.8 & 3.7 & 3.4 & 4.1 & 8.4 & 11.7 & 15.2 & 16.2 \\
\hline & Chungbuk & - & - & 0.9 & 1.1 & 1.7 & 2.4 & 3.9 & 5.9 & 7.0 & 9.0 & 13.9 \\
\hline & Chungnam & - & - & 1.5 & 1.2 & 2.8 & 3.3 & 4.4 & 6.6 & 9.3 & 8.4 & 12.1 \\
\hline & Gyeongnam & - & - & 0.6 & 1.8 & 1.3 & 3.2 & 4.7 & 6.2 & 8.2 & 9.7 & 11.5 \\
\hline & Jeju & - & - & 1.4 & 2.5 & 1.9 & 3.5 & 5.4 & 8.5 & 11.7 & 17.9 & 17.0 \\
\hline & Disparity (max-min) & - & - & 2.5 & 4.5 & 6.3 & 11.2 & 14.6 & 17.2 & 23.6 & 17.3 & 23.5 \\
\hline \multirow{7}{*}{$\begin{array}{l}\text { Rate of ROSC before } \\
\text { hospital arrival (\%) }\end{array}$} & Total & 0.9 & 1.1 & 1.2 & 1.9 & 1.8 & 3.1 & 3.5 & 3.7 & 4.2 & 5.1 & 6.9 \\
\hline & Seoul & 1.2 & 1.3 & 1.5 & 2.6 & 2.0 & 2.8 & 4.1 & 4.5 & 5.0 & 6.2 & 7.7 \\
\hline & Busan & 0.9 & 0.8 & 1.7 & 2.3 & 2.0 & 3.0 & 4.6 & 5.1 & 5.6 & 6.7 & 10.2 \\
\hline & Daegu & 1.3 & 0.4 & 0.6 & 1.6 & 2.2 & 3.6 & 3.3 & 4.7 & 4.3 & 5.6 & 9.0 \\
\hline & Incheon & 0.3 & 1.1 & 1.9 & 1.8 & 2.0 & 3.8 & 3.3 & 3.8 & 5.0 & 5.5 & 7.5 \\
\hline & Gwangju & 0.2 & 0.8 & 2.0 & 1.6 & 2.4 & 5.2 & 3.8 & 4.2 & 5.6 & 4.5 & 9.4 \\
\hline & Daejeon & 0.4 & 1.1 & 1.4 & 1.2 & 3.0 & 3.9 & 3.1 & 5.6 & 4.8 & 4.3 & 7.3 \\
\hline
\end{tabular}


Table 1. Continued

\begin{tabular}{|c|c|c|c|c|c|c|c|c|c|c|c|c|}
\hline Index & Value & 2006 & 2007 & 2008 & 2009 & 2010 & 2011 & 2012 & 2013 & 2014 & 2015 & 2016 \\
\hline & Chungnam & 0.8 & 1.4 & 0.4 & 1.5 & 1.0 & 4.4 & 3.0 & 2.5 & 2.3 & 2.6 & 5.8 \\
\hline & Jeonbuk & 0.6 & 0.6 & 0.5 & 1.5 & 1.6 & 4.1 & 3.7 & 4.7 & 4.4 & 4.7 & 4.1 \\
\hline & Jeonnam & 0.7 & 1.6 & 1.0 & 1.6 & 1.8 & 2.5 & 2.7 & 2.3 & 2.4 & 4.1 & 3.0 \\
\hline & Gyeongbuk & 0.9 & 1.0 & 1.1 & 1.5 & 0.9 & 1.7 & 2.4 & 2.7 & 2.2 & 3.6 & 4.4 \\
\hline & Gyeongnam & 0.4 & 0.4 & 0.8 & 1.8 & 1.4 & 2.5 & 2.5 & 2.9 & 3.2 & 4.6 & 5.1 \\
\hline & Jeju & 0.7 & 1.2 & 1.2 & 2.6 & 1.6 & 4.9 & 5.5 & 3.9 & 3.3 & 5.1 & 5.1 \\
\hline & Disparity (max-min) & 1.6 & 1.2 & 1.6 & 1.4 & 2.5 & 3.5 & 3.4 & 3.3 & 4.2 & 4.9 & 12.3 \\
\hline \multirow[t]{19}{*}{ Survival rate $(\%)$} & Total & 2.3 & 2.6 & 2.5 & 3.3 & 3.3 & 4.1 & 4.4 & 4.8 & 4.8 & 6.3 & 7.6 \\
\hline & Seoul & 3.9 & 5.0 & 4.9 & 6.4 & 6.3 & 8.3 & 8.8 & 9.2 & 8.6 & 9.8 & 11.4 \\
\hline & Busan & 0.6 & 2.0 & 2.0 & 3.5 & 3.6 & 4.5 & 4.4 & 5.3 & 5.7 & 7.6 & 9.1 \\
\hline & Daegu & 2.2 & 1.3 & 1.7 & 2.4 & 2.4 & 3.7 & 4.7 & 6.1 & 5.4 & 5.1 & 7.0 \\
\hline & Incheon & 4.3 & 3.9 & 4.5 & 4.1 & 4.0 & 5.8 & 5.3 & 6.7 & 5.8 & 7.2 & 8.2 \\
\hline & Gwangju & 3.2 & 2.0 & 3.1 & 4.6 & 4.0 & 4.1 & 2.9 & 4.6 & 3.9 & 7.6 & 9.2 \\
\hline & Daejeon & 4.4 & 3.9 & 4.2 & 4.2 & 8.1 & 6.6 & 6.8 & 7.9 & 7.3 & 7.1 & 8.4 \\
\hline & Ulsan & 1.4 & 1.9 & 0.5 & 2.7 & 4.4 & 3.3 & 3.8 & 3.0 & 4.5 & 6.4 & 10.9 \\
\hline & Sejong & - & - & - & - & - & - & 5.3 & 2.6 & 3.7 & 2.2 & 11.9 \\
\hline & Gyeonggi & 3.0 & 3.4 & 2.8 & 3.7 & 3.3 & 4.0 & 4.8 & 4.8 & 5.7 & 6.8 & 8.2 \\
\hline & Gangwon & 3.2 & 2.4 & 1.9 & 2.9 & 4.1 & 3.1 & 3.6 & 3.8 & 4.5 & 4.7 & 6.1 \\
\hline & Chungbuk & 1.7 & 1.2 & 1.4 & 2.0 & 1.5 & 3.0 & 2.5 & 3.8 & 2.8 & 3.9 & 5.3 \\
\hline & Chungnam & 0.3 & 0.7 & 0.7 & 1.2 & 0.7 & 2.5 & 2.6 & 1.9 & 1.9 & 3.5 & 6.1 \\
\hline & Jeonbuk & 1.1 & 2.4 & 1.2 & 1.5 & 2.1 & 3.0 & 2.9 & 4.4 & 3.2 & 4.8 & 5.1 \\
\hline & Jeonnam & 0.9 & 1.4 & 1.4 & 1.4 & 1.2 & 1.7 & 1.6 & 1.2 & 1.1 & 4.4 & 4.7 \\
\hline & Gyeongbuk & 0.7 & 0.6 & 1.1 & 1.3 & 1.1 & 1.6 & 1.4 & 1.8 & 1.3 & 3.4 & 4.3 \\
\hline & Gyeongnam & 0.2 & 0.5 & 1.9 & 1.8 & 1.1 & 1.6 & 2.1 & 2.3 & 2.6 & 4.5 & 5.5 \\
\hline & Jeju & 1.4 & 2.7 & 1.8 & 3.2 & 2.8 & 3.5 & 4.6 & 3.6 & 3.6 & 7.4 & 6.2 \\
\hline & Disparity (max-min) & 4.2 & 4.5 & 4.4 & 5.2 & 7.4 & 6.7 & 7.4 & 8.0 & 7.5 & 7.6 & 7.6 \\
\hline \multirow{19}{*}{$\begin{array}{l}\text { Neurological } \\
\text { recovery rate (\%) }\end{array}$} & Total & 0.6 & 0.7 & 0.8 & 1.0 & 0.9 & 1.6 & 1.8 & 2.3 & 2.7 & 3.6 & 4.2 \\
\hline & Seoul & 0.8 & 1.6 & 1.5 & 1.7 & 1.6 & 2.9 & 3.3 & 3.9 & 4.9 & 5.8 & 6.0 \\
\hline & Busan & 0.0 & 0.4 & 0.3 & 1.2 & 0.8 & 1.9 & 2.2 & 3.0 & 3.1 & 4.6 & 5.3 \\
\hline & Daegu & 1.1 & 0.0 & 0.9 & 1.1 & 1.2 & 2.2 & 2.3 & 3.9 & 3.7 & 3.7 & 5.4 \\
\hline & Incheon & 1.0 & 1.1 & 0.8 & 1.3 & 1.4 & 2.1 & 2.2 & 3.0 & 3.8 & 3.8 & 4.5 \\
\hline & Gwangju & 0.2 & 0.6 & 0.9 & 1.1 & 0.6 & 2.1 & 1.6 & 2.2 & 2.2 & 3.7 & 4.8 \\
\hline & Daejeon & 1.6 & 1.5 & 1.4 & 0.3 & 1.4 & 2.9 & 1.9 & 3.8 & 3.3 & 3.9 & 4.0 \\
\hline & Ulsan & 0.6 & 1.1 & 0.3 & 1.0 & 2.1 & 0.9 & 2.3 & 1.5 & 2.6 & 4.0 & 6.2 \\
\hline & Sejong & - & - & - & - & - & - & 1.8 & 2.6 & 3.7 & 0.0 & 9.3 \\
\hline & Gyeonggi & 0.8 & 0.8 & 0.9 & 0.9 & 1.0 & 1.3 & 2.1 & 2.4 & 2.8 & 3.8 & 4.6 \\
\hline & Gangwon & 1.4 & 0.9 & 0.6 & 1.7 & 1.4 & 1.1 & 1.0 & 2.1 & 2.5 & 2.5 & 2.5 \\
\hline & Chungbuk & 0.4 & 0.3 & 0.3 & 1.0 & 0.6 & 1.0 & 0.7 & 1.4 & 1.8 & 2.5 & 3.1 \\
\hline & Chungnam & 0.0 & 0.3 & 0.0 & 0.3 & 0.1 & 1.1 & 1.2 & 0.8 & 0.9 & 1.9 & 2.9 \\
\hline & Jeonbuk & 0.2 & 0.1 & 0.3 & 0.4 & 0.4 & 1.4 & 1.2 & 2.0 & 2.2 & 2.7 & 3.3 \\
\hline & Jeonnam & 0.2 & 0.1 & 0.7 & 0.2 & 0.3 & 0.4 & 0.5 & 0.6 & 0.4 & 2.3 & 2.0 \\
\hline & Gyeongbuk & 0.2 & 0.1 & 0.3 & 0.6 & 0.5 & 0.6 & 0.9 & 0.9 & 0.8 & 2.3 & 2.7 \\
\hline & Gyeongnam & 0.1 & 0.2 & 0.5 & 0.5 & 0.4 & 0.7 & 1.1 & 1.5 & 1.8 & 2.7 & 3.1 \\
\hline & Јејu & 0.5 & 1.4 & 0.9 & 1.7 & 1.2 & 2.8 & 2.7 & 2.0 & 2.2 & 4.6 & 3.7 \\
\hline & Disparity (max-min) & 1.6 & 1.6 & 1.5 & 1.5 & 2.0 & 2.5 & 2.8 & 3.3 & 4.5 & 5.8 & 7.3 \\
\hline
\end{tabular}

Unit: \%, \%p.

CPR, cardiopulmonary resuscitation; ROSC, return of spontaneous circulation; -, null value.

and trends toward increasing regional disparities were observed in the rates of survival and neurological recovery, an indicator of treatment results. These disparities also increased with respect to the type of city. For example, Seoul, which is unique among the various city types, exhibited better overall performances and magnitudes of improvement with respect to survival and neurological 
Table 2. Results of sudden cardiac arrest main indices and their disparities among city types, 2006-2016

\begin{tabular}{|c|c|c|c|c|c|c|c|c|c|c|c|c|c|}
\hline Index & City type & & 2006 & 2007 & 2008 & 2009 & 2010 & 2011 & 2012 & 2013 & 2014 & 2015 & 2016 \\
\hline \multirow{6}{*}{$\begin{array}{l}\text { Rate of CPR } \\
\text { performed by } \\
\text { layperson }(\%)\end{array}$} & \multicolumn{2}{|l|}{ Local districts of Seoul metropolitan city } & - & - & $3.1^{\text {a) }}$ & $5.5^{\text {a) }}$ & $7.3^{\text {a) }}$ & $12.3^{\mathrm{a})}$ & $17.4^{\mathrm{a})}$ & $19.8^{\mathrm{a})}$ & $26.2^{\text {a) }}$ & $23.6^{\text {a) }}$ & $29.6^{\text {a) }}$ \\
\hline & \multicolumn{2}{|l|}{ Local districts of Metropolitan city) } & - & - & 1.9 & 2.8 & 3.5 & 5.0 & 6.4 & 8.8 & 12.8 & 15.2 & 17.7 \\
\hline & \multicolumn{2}{|l|}{ Cities with less than 300,000 people } & - & - & 1.7 & $1.1^{\mathrm{c}}$ & 1.7 & 2.3 & 4.3 & 5.5 & 10.4 & 11.4 & 15.9 \\
\hline & \multicolumn{2}{|l|}{ Urban rural consolidated cities } & - & - & 1.3 & 1.5 & 2.0 & 2.7 & 3.4 & 5.4 & 7.3 & 9.0 & 10.2 \\
\hline & \multicolumn{2}{|l|}{ Counties } & - & - & $1.1^{c)}$ & 1.2 & $1.4^{c)}$ & $1.8^{\mathrm{c})}$ & $2.8^{\mathrm{c})}$ & $4.4^{\mathrm{c})}$ & $6.4^{\mathrm{c})}$ & $7.7^{\mathrm{c})}$ & $9.5^{c)}$ \\
\hline & \multicolumn{2}{|l|}{ Disparity (max-min) } & - & - & 2.0 & 4.4 & 5.9 & 10.5 & 14.6 & 15.4 & 19.8 & 15.9 & 20.1 \\
\hline \multirow{6}{*}{$\begin{array}{l}\text { ROSC before } \\
\text { hospital arrival } \\
(\%)\end{array}$} & \multicolumn{2}{|l|}{ Local districts of Seoul metropolitan city } & $1.2^{\mathrm{a})}$ & 1.3 & $1.5^{\mathrm{a})}$ & $2.6^{a)}$ & 2.0 & 2.8 & $4.1^{\text {a) }}$ & $4.5^{\text {a) }}$ & $5.0^{a)}$ & $6.2^{\text {a) }}$ & 7.7 \\
\hline & \multicolumn{2}{|l|}{ Local districts of Metropolitan city ${ }^{b)}$} & 0.9 & 1.0 & 1.3 & 1.9 & 1.9 & $3.4^{\text {a) }}$ & 3.6 & 4.1 & 4.5 & 5.5 & $8.2^{\mathrm{a})}$ \\
\hline & \multicolumn{2}{|l|}{ Cities with less than 300,000 people } & 0.9 & $1.5^{\text {a) }}$ & $0.9^{c)}$ & 1.8 & $2.1^{\text {a) }}$ & $2.2^{\mathrm{c})}$ & 3.7 & 3.0 & 4.2 & 5.6 & 6.1 \\
\hline & \multicolumn{2}{|l|}{ Urban rural consolidated cities } & 0.9 & $1.0^{c)}$ & 1.0 & 1.7 & 1.6 & 2.9 & $2.9^{c)}$ & 2.9 & 3.4 & 3.9 & 4.9 \\
\hline & \multicolumn{2}{|l|}{ Counties } & $0.7^{c)}$ & $1.0^{c)}$ & $0.9^{c)}$ & $1.6^{c)}$ & $1.2^{\mathrm{c})}$ & 3.0 & 3.0 & $2.8^{\mathrm{c})}$ & $3.1^{\mathrm{c})}$ & $3.6^{c)}$ & $4.7^{c)}$ \\
\hline & \multicolumn{2}{|l|}{ Disparity (max-min) } & 0.5 & 0.5 & 0.6 & 1.0 & 0.9 & 1.2 & 1.2 & 1.7 & 1.9 & 2.6 & 3.5 \\
\hline \multirow[t]{11}{*}{ Survival rate (\%) } & \multirow{2}{*}{$\begin{array}{l}\text { Local districts of Seoul metropolitan } \\
\text { city }\end{array}$} & $\mathrm{CR}$ & $3.9^{\mathrm{a})}$ & $5.0^{a)}$ & $4.9^{\mathrm{a})}$ & $6.4^{a)}$ & $6.3^{a)}$ & $8.3^{\mathrm{a})}$ & $8.8^{\mathrm{a})}$ & $9.2^{\mathrm{a})}$ & $8.6^{\text {a) }}$ & $9.8^{\text {a) }}$ & $11.4^{\mathrm{a})}$ \\
\hline & & Std & $4.0^{\mathrm{a})}$ & $5.0^{a)}$ & $4.9^{\mathrm{a})}$ & $6.5^{\text {a) }}$ & $6.5^{a)}$ & $8.5^{\text {a) }}$ & $9.3^{\mathrm{a})}$ & $9.9^{\mathrm{a})}$ & $9.8^{\text {a) }}$ & $11.2^{\mathrm{a})}$ & $12.9^{\mathrm{a})}$ \\
\hline & \multirow[t]{2}{*}{ Local districts of Metropolitan city ${ }^{b)}$} & $\mathrm{CR}$ & 2.9 & 3.0 & 2.9 & 3.8 & 3.8 & 4.6 & 5.0 & 5.7 & 5.6 & 6.8 & 8.4 \\
\hline & & Std & 2.8 & 2.9 & 2.9 & 3.8 & 3.9 & 4.7 & 5.2 & 6.0 & 6.1 & 7.5 & 9.2 \\
\hline & \multirow[t]{2}{*}{ Cities with less than 300,000 people } & $\mathrm{CR}$ & 1.3 & 1.7 & 2.3 & 3.6 & 3.1 & 3.8 & 3.4 & 3.9 & 4.2 & 6.8 & 7.4 \\
\hline & & Std & 1.3 & 1.6 & 2.4 & 3.6 & 2.9 & 3.7 & 3.5 & 4.1 & 4.6 & 8.6 & 8.5 \\
\hline & \multirow[t]{2}{*}{ Urban rural consolidated cities } & $\mathrm{CR}$ & 1.0 & 1.1 & 1.4 & 1.6 & 1.6 & 1.8 & 2.2 & 2.1 & 2.2 & 4.3 & 5.5 \\
\hline & & Std & 1.0 & 1.1 & 1.4 & 1.7 & 1.6 & 2.0 & 2.4 & 2.3 & 2.4 & 5.1 & 6.6 \\
\hline & \multirow[t]{2}{*}{ Counties } & $\mathrm{CR}$ & $0.3^{\text {c) }}$ & $0.6^{c)}$ & $0.5^{c)}$ & $0.7^{c)}$ & $0.7^{\mathrm{c})}$ & $1.4^{c)}$ & $1.3^{\mathrm{c}}$ & $1.3^{\mathrm{c})}$ & $1.3^{\mathrm{c})}$ & $3.0^{\mathrm{c})}$ & $3.9^{c)}$ \\
\hline & & Std & $0.3^{\mathrm{c}}$ & $0.6^{c)}$ & $0.5^{\mathrm{c})}$ & $0.8^{\mathrm{c})}$ & $0.8^{\mathrm{c})}$ & $1.6^{\mathrm{c})}$ & $1.4^{\mathrm{cl}}$ & $1.6^{\mathrm{c})}$ & $1.5^{\mathrm{c})}$ & $3.7^{\mathrm{c})}$ & $4.6^{c)}$ \\
\hline & \multicolumn{2}{|l|}{ Disparity (max-min) } & 3.6 & 4.4 & 4.4 & 5.7 & 5.6 & 6.9 & 7.5 & 7.9 & 7.3 & 6.8 & 7.5 \\
\hline \multirow{11}{*}{$\begin{array}{l}\text { Neurological } \\
\text { recovery rate } \\
(\%)\end{array}$} & \multirow{2}{*}{$\begin{array}{l}\text { Local districts of Seoul metropolitan } \\
\text { city }\end{array}$} & $\mathrm{CR}$ & $0.8^{\mathrm{a})}$ & $1.6^{a)}$ & $1.5^{\mathrm{a})}$ & $1.7^{\mathrm{a})}$ & $1.6^{a)}$ & $2.9^{\mathrm{a})}$ & $3.3^{\mathrm{a})}$ & $3.9^{\mathrm{a})}$ & $4.9^{\mathrm{a})}$ & $5.8^{\mathrm{a})}$ & $6.0^{\mathrm{a})}$ \\
\hline & & Std & $0.9^{\mathrm{a})}$ & $1.6^{a)}$ & $1.6^{a)}$ & $1.7^{\mathrm{a})}$ & $1.6^{a)}$ & $3.0^{\mathrm{a})}$ & $3.5^{\text {a) }}$ & $4.4^{\mathrm{a})}$ & $5.9^{\text {a) }}$ & $6.8^{\mathrm{a})}$ & $7.1^{\text {a) }}$ \\
\hline & \multirow[t]{2}{*}{ Local districts of Metropolitan city } & $\mathrm{CR}$ & 0.7 & 0.8 & 0.8 & 1.1 & 1.1 & 1.8 & 2.2 & 2.8 & 3.2 & 4.0 & 4.9 \\
\hline & & Std & 0.7 & 0.8 & 0.8 & 1.1 & 1.1 & 1.9 & 2.4 & 3.0 & 3.4 & 4.5 & 5.5 \\
\hline & \multirow[t]{2}{*}{ Cities with less than 300,000 people } & $\mathrm{CR}$ & 0.7 & 0.6 & 1.2 & 1.1 & 1.1 & 1.3 & 1.2 & 1.8 & 1.8 & 3.3 & 3.2 \\
\hline & & Std & 0.8 & 0.5 & 1.3 & 1.1 & 1.0 & 1.2 & 1.2 & 1.9 & 2.1 & 4.6 & 4.0 \\
\hline & Urban rural consolidated cities & $\mathrm{CR}$ & 0.3 & 0.3 & 0.4 & 0.6 & 0.6 & 0.7 & 1.0 & 1.1 & 1.3 & 2.2 & 2.7 \\
\hline & & Std & 0.4 & 0.3 & 0.4 & 0.7 & 0.6 & 0.8 & 1.2 & 1.3 & 1.5 & 2.6 & 3.5 \\
\hline & Counties & $\mathrm{CR}$ & $0.1^{\mathrm{c}}$ & $0.1^{c)}$ & $0.2^{\mathrm{c})}$ & $0.3^{c)}$ & $0.3^{\mathrm{c})}$ & $0.6^{c)}$ & $0.5^{\mathrm{c}}$ & $0.8^{\mathrm{c})}$ & $0.8^{\mathrm{c})}$ & $1.9^{c)}$ & $2.1^{c)}$ \\
\hline & & Std & $0.1^{\mathrm{c}}$ & $0.1^{c)}$ & $0.2^{\mathrm{c})}$ & $0.4^{c)}$ & $0.4^{c)}$ & $0.7^{c)}$ & $0.6^{\mathrm{cl}}$ & $1.0^{c)}$ & $1.1^{\mathrm{cl}}$ & $2.5^{\mathrm{c})}$ & $2.6^{c)}$ \\
\hline & Disparity (max-min) & & 0.7 & 1.5 & 1.3 & 1.4 & 1.3 & 2.3 & 2.8 & 3.1 & 4.1 & 3.9 & 3.9 \\
\hline
\end{tabular}

$\mathrm{CPR}$, cardiopulmonary resuscitation; ROSC, return of spontaneous circulation; -, null value; $\mathrm{CR}$, crude rate; Std, standardized rate.

a) Region with the highest values in the year. ${ }^{b}$ Local districts of Metropolitan cities, cites with more than 300,000 population, or local districts of cities with more than 500,000 population or 'Gu' of 'Si' with more than 500,000 population. ${ }^{c}$ Region with the lowest values in the year.

recovery, compared to other city types (Tables 1, 2).

The timely administration of treatment is crucial during a sudden cardiac arrest, and patient prognosis improves only in the presence of effective and organic flow between different strata (i.e., regional society, emergency response, and hospital stages). Therefore, the survival rate following sudden cardiac arrest can be considered an indicator of a nation's entire emergency medical system. The introduction of a sudden cardiac arrest survey program in 2008 enabled the objective assessment of issues related to sudden cardiac arrest and the generation of comparable regional statistics and individualized evidence-based policies. From this, we could confirm that overall survival of sudden cardiac ar- rests improved nationwide while regional disparities widened.

According to a recent study, the survival rate among cases of sudden cardiac arrest increased by 1.4 -fold as the proportion of the population educated in CPR increased by $10 \%$. $^{1}$ However, a community health survey (citizen health statistics) conducted annually by 254 community health centers as stated by community health law found that although the proportion of the overall population educated in CPR improved, regional disparities were apparent. ${ }^{2}$ Accordingly, central and local governments should focus not only on improving overall survival nationwide, but should also reinforce policies that aim to reduce these widening gaps. Finally, we hope that the regional statistics yielded by the Sudden Cardi- 
ac Arrest Survey will promote initiatives aimed at improving the survival of community-dwelling patients who experience sudden cardiac arrest.

\section{CONFLICT OF INTEREST}

No potential conflict of interest relevant to this article was reported.

\section{REFERENCES}

1. Ro YS, Shin SD, Song KJ, et al. Public awareness and self-efficacy of cardiopulmonary resuscitation in communities and outcomes of out-of-hospital cardiac arrest: a multi-level analysis. Resuscitation 2016;102:17-24.

2. Korea Centers for Disease Control and Prevention. 2008-2016 Community health statistics at a glance. Cheongju: Korea Centers for Disease Control and Prevention; 2017. 